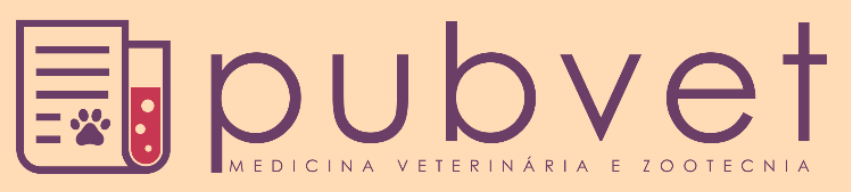

https://doi.org/10.31533/pubvet.v15n10a929.1-16

\title{
Hipercoagulabilidade secundária ao hiperadrenocorticismo em cães: Revisão
}

\author{
Gabriela Barbosa de Almeida $^{1 *}$, Mariana Santos de Miranda ${ }^{2} \bullet$, Douglas Segalla Caragelasco ${ }^{2} \bullet$, \\ Michele Andrade de Barros $^{2}$ o
}

${ }^{1}$ Aluna da graduação da Pontifícia Universidade Católica de Campinas, Departamento de Medicina Veterinária, Campinas, São Paulo, Brasil. ${ }^{2}$ Professor (a) da Pontifícia Universidade Católica de Campinas, Departamento de Medicina Veterinária, Campinas, São Paulo, Brasil. *Autor para correspondência, E-mail: gabibalmeida23@outlook.com

Resumo. O Hiperadrenocorticismo ou Síndrome de Cushing é uma endocrinopatia caracterizada pela elevada concentração de cortisol na circulação. Esse hormônio é responsável por inúmeras alterações no organismo, dentre elas o aumento da coagulação que contribui para a formação de trombos. Essa complicação é considerada fatal, nesse sentido, o objetivo do presente estudo foi, através de revisões literárias e acadêmicas de artigos científicos, compreender a fisiopatologia da hipercoagulabilidade secundária ao hiperadrenocorticismo, além de identificar métodos diagnósticos para tais anomalias e seus possíveis tratamentos. Numerosas possibilidades foram encontradas para explicar o aumento da coagulação na síndrome de Cushing, no entanto, todas as teorias citadas pelos autores ainda são pouco compreendidas. O diagnóstico da Síndrome de Cushing pode ser realizado por diversos métodos, sendo o mais utilizado o exame de supressão por dose baixa de dexametasona. Já a hipercoagulabilidade pode ser identificada através da tromboelastrografia ou da tromboelastometria rotacional, esses testes avaliam a hemostasia de maneira íntegra. Além desses exames, outros métodos diagnósticos também podem auxiliar na avaliação da situação hemostática do animal, como a contagem plaquetária, a mensuração dos fatores de coagulação, a concentração de fibrinogênio, entre outros. $\mathrm{O}$ tratamento do hiperadrenocorticismo consiste basicamente na administração de trilostano ou mitotano. No entanto, o controle desse hormônio não garante melhora significativa do estado hipercoagulável presente no paciente, uma vez que o cortisol possui efeito prolongado no organismo e, dependendo da posologia instituída, há um intervalo de tempo sem ação do medicamento. Desse modo, essa complicação deve ser tratada ou quando os testes apresentarem sinais de hipercoagulabilidade ou, quando o animal possuir uma afecção concomitante que predisponha a formação de trombos, estabelecendo, então, uma terapia com anticoagulantes.

Palavras chave: Hiperadrenocorticismo, hipercoagulabilidade, síndrome de cushing

\section{Secondary hypercoagulability to hyperadrenocorticism in dogs: Review}

\begin{abstract}
Hyperadrenocorticism or Cushing's Syndrome is an endocrinopathy characterized by a high concentration of cortisol in the circulation. This hormone is responsible for numerous changes in the body, including increased clotting that contributes to the formation of thrombi. This complication is considered highly fatal, in this sense, the objective of the present study was to understand, through literature and academic review of scientific papers, the pathophysiology of hypercoagulability secondary to hyperadrenocorticism, in addition to identifying diagnostic methods for such anomalies and their possible treatments. Numerous possibilities have been found to explain the increase in coagulation, however, all the theories cited by the authors are still poorly understood. The diagnosis of Cushing's Syndrome can be made by several methods, the most used being the low dose dexamethasone suppression test. Hypercoagulability, on the other hand, may be identified through thromboelastrography or
\end{abstract}


rotational thromboelastometry, these tests may fully evaluate hemostasis. In addition to these tests, other diagnostic methods can also assist in the assessment of the animal's hemostatic situation, such as platelet count, measurement of coagulation factors, fibrinogen concentration, among others. The treatment of hyperadrenocorticism basically consists of the administration of trilostane or mitotane. Nevertheless, the control of this hormone does not guarantee a significant improvement in the hypercoagulable state present in the patient, since cortisol has a prolonged effect on the body and depending on the dosage established for a period of time without action of the medication. Hence, this complication must be treated when the tests show signs of hypercoagulability or when the animal has a concomitant condition that predisposes to the formation of thrombi, and then establishes anticoagulant therapy.

Keywords: Hyperadrenocorticism, hypercoagulability, cushing's syndrome

\section{Introdução}

O hiperadrenocorticismo ou Síndrome de Cushing, é uma das principais endocrinopatias diagnosticadas na espécie canina (Jericó et al., 2015). Essa afecção hormonal afeta comumente cães de meia idade e, é caracterizada pela liberação excessiva do hormônio cortisol na circulação sanguínea. Esse excesso de cortisol é responsável por inúmeras alterações físicas e químicas em diferentes sistemas do organismo animal (Feldman et al., 2014; Peterson, 1984).

A hipercoagulabilidade pode ocorrer secundariamente a muitas doenças sistêmicas que afetam os animais, dentre elas o hiperadrenocorticismo (Nelson \& Couto, 2015). Esse aumento da coagulação pode estar relacionado com a ocorrência de eventos trombóticos nos cães com hipercortisolismo, principalmente, o tromboembolismo pulmonar que é uma complicação em latência nessa endocrinopatia. Ele consiste na obstrução de vasos pulmonares por um trombo, que é formado através das alterações hemostáticas desenvolvidas pela ação hormonal (Jericó et al., 2015; Nelson \& Couto, 2015; Waki et al., 2010).

Nesse sentido, o tromboembolismo pulmonar é uma das poucas complicações do hiperadrenocorticismo que pode ser fatal, sendo assim, o prognóstico da afecção quando associada a esse agravante é considerado de reservado a grave (Feldman et al., 2014; Nelson \& Couto, 2015). Além do tromboêmbolo no pulmão, o aumento da coagulação pode promover tromboses venosas e arteriais que afetam outros órgãos (Marco et al., 2012; Waki et al., 2010).

O objetivo do presente estudo foi, portanto, compreender pelas revisões literárias e acadêmicas de artigos científicos a fisiopatologia da hipercoagulabilidade desenvolvida pelo hipercortisolismo, bem como auxiliar na realização do diagnóstico precoce de ambas as anormalidades. Por fim, contribuir na escolha individual do melhor tratamento a ser seguido, visto que as consequências da hipercoagulabilidade podem ser fatais quando não solucionadas corretamente.

\section{Hiperadrenocorticismo}

O hiperadrenocorticismo (HAC) ou síndrome de Cushing, é caracterizado pelo elevado valor de cortisol circulante e, consequentemente, pelas variedades de manifestações clínicas e laboratoriais que promovem (Peterson, 1984). É considerada a endocrinopatia mais diagnostica em cães, principalmente, os que possuem idade acima de seis anos (Feldman et al., 2015). Não apresenta predisposição sexual nos casos de HAC dependente da hipófise, entretanto, nos casos em que a síndrome ocorre em dependência da adrenal há uma prevalência de 70 a $75 \%$ em fêmeas (Peterson, 1984). As principais raças acometidas são: Poodle, Dachshund, raças Terrier, Pastores-alemães, Beagles, Boxer e Labradores (Marco, 2015; Feldman et al., 2015; Nelson \& Couto, 2015; Peterson, 1984).

\section{Anatofisiologia da adrenal}

As glândulas adrenais estão localizadas craniomedialmente aos rins e são divididas em córtex e medula, essas duas regiões são distintas estrutural e funcionalmente. O córtex da adrenal é designado por uma coloração mais clara com estrias radiais e se origina a partir das células mesenquimais do mesoderma. Funcionalmente, o córtex é responsável pela produção dos hormônios mineralocorticoides e os glicocorticoides, respectivamente, aldosterona e cortisol, uma parcela de 5\% dessa área é designada 
a produção de alguns hormônios sexuais, que auxiliam no desenvolvimento dos órgãos genitais masculinos (Köning \& Liebich, 2011; Marco, 2015).

A região medular dessa glândula possui uma coloração mais escura e se origina de células ectodérmicas. Essa região é encarregada de secretar os neurotransmissores adrenalina e noradrenalina, as quais possuem ação no sistema nervoso simpático e na pressão sanguínea (Köning \& Liebich, 2011; Rijnberk \& Kooistra, 2010).

O controle da liberação do hormônio cortisol é realizado pelo eixo corticotrófico, conforme Figura 1. Segundo Marco (2015) esse eixo consiste na liberação pelo hipotálamo do hormônio corticotropina (CRH), que é secretado devido a vários estímulos, como o estresse. $\mathrm{O}$ CRH possui receptor $\mathrm{CRH} 1$ na hipófise, estimulando a liberação do hormônio adrenocorticotrófico (ACTH), o qual irá incentivar a adrenal a sintetizar e secretar o cortisol. O cortisol, por sua vez, completa o ciclo e exerce um efeito inibitório nesse eixo quando se apresenta elevado na circulação (Feldman et al., 2015). Ou seja, é realizado um feedback negativo que diminui a produção de CRH e de ACTH e, com isso, há o controle da liberação de cortisol no sangue, essa condição acontece apenas em animais saudáveis (Marco, 2015).

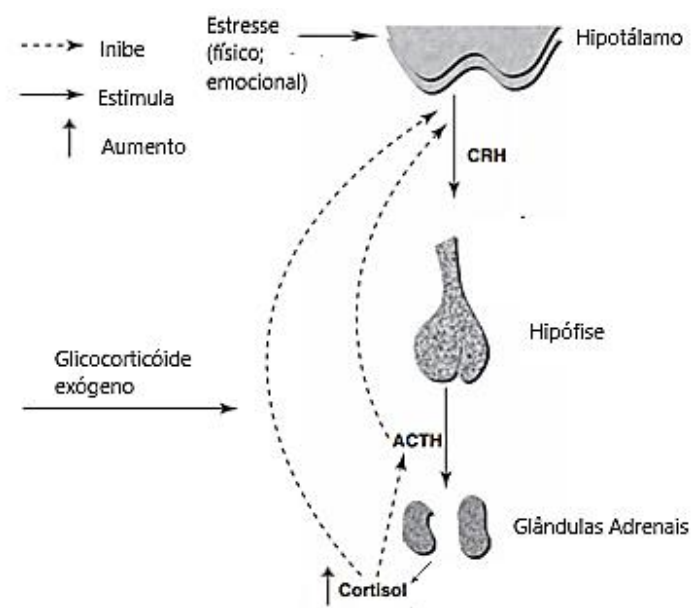

Figura 1. Eixo corticotrófico. Esquema que exemplifica o eixo corticotrófico, indicando os estímulos desencadeados. O hipotálamo, por estímulo externo, libera o hormônio CRH, o qual estimula a hipófise/pituitária a liberar ACTH. O ACTH, por sua vez incita a adrenal a produzir e secretar cortisol. O cortisol, quando em excesso no sangue, realiza um feedback negativo que diminui a produção de $\mathrm{CRH}$ e $\mathrm{ACTH}$, inibindo todo o eixo. Fonte: Adaptado Feldman et al. (2015, p. 379).

\section{Classificação do hiperadrenocorticismo}

O HAC pode ser classificado como: dependente da hipófise, dependente da adrenal ou iatrogênico (Nelson \& Couto, 2015).

\subsection{Hiperadrenocorticismo hipófise-dependente}

O HAC dependente da hipófise é o mais comum, acometendo 80-85\% dos animais que apresentam a síndrome (Feldman et al., 2015; Nelson \& Couto, 2015). Nessa classificação, é encontrado, na maioria dos casos, um adenoma na hipófise que aumenta a produção e liberação de ACTH. O excesso desse hormônio causa uma hiperplasia adrenocortical bilateral, de acordo com a Figura 2, e, com isso, secreção excessiva de cortisol (Nelson \& Couto, 2015). Normalmente, 75\% dos cães que possuem o HAC dependente da hipófise pesam menos de $20 \mathrm{~kg}$ (Feldman et al., 2015; Nelson \& Couto, 2015).

\subsection{Hiperadrenocorticismo adrenal-dependente}

O hipercortisolismo dependente da adrenal, a qual apresenta um adenoma ou um carcinoma na glândula uni ou bilateralmente, representa 15-20\% dos casos (Nelson \& Couto, 2015). Esses tumores secretam quantidades excessivas de cortisol e, com isso, causam um desequilíbrio no eixo corticotrófico, pois o excesso de cortisol suprime a liberação de $\mathrm{CRH}$ e $\mathrm{ACTH}$, inibindo todo o eixo. Essa inibição 
crônica promove a atrofia da glândula que não está com a neoplasia, em exceção de tumores bilaterais, conforme a Figura 2. (Marco, 2015; Nelson \& Couto, 2015). Esse quadro, é comum em cães que pesam mais de $20 \mathrm{~kg}$, aproximadamente, $50 \%$ dos animais nessa situação se encontram nesse escore corporal (Feldman et al., 2015; Nelson \& Couto, 2015).
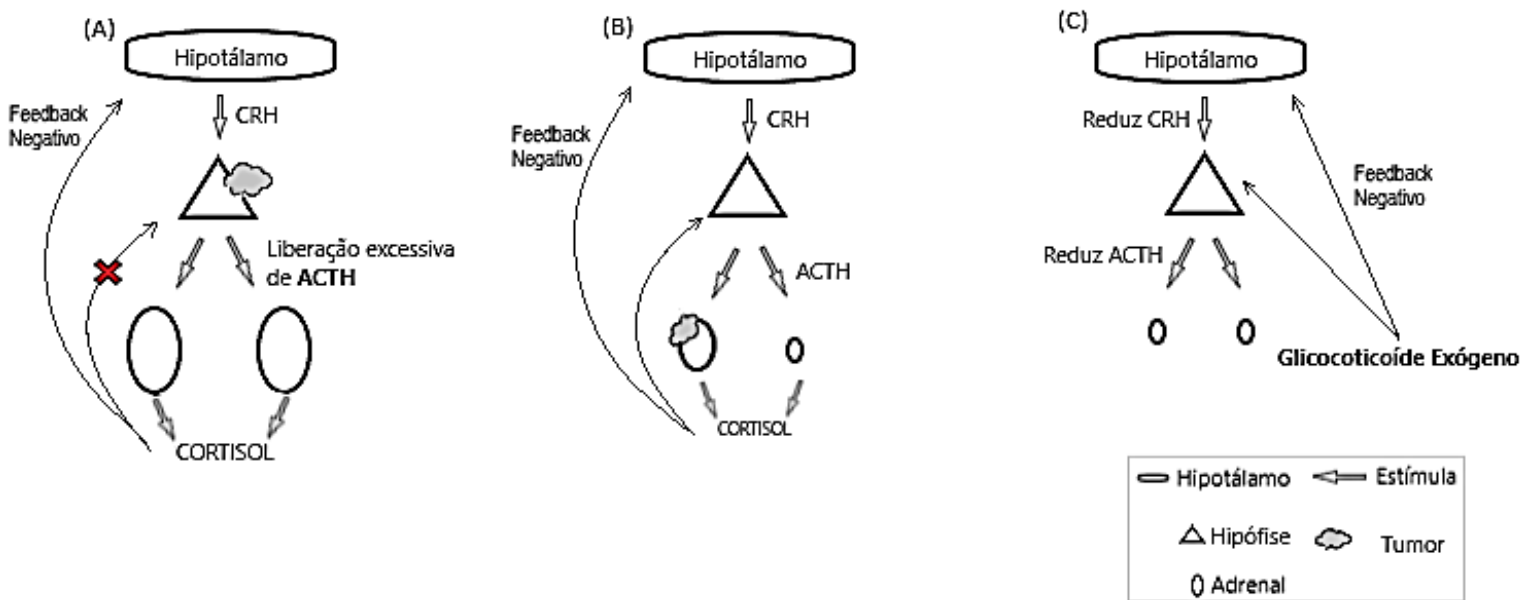

Figura 2. Esquema HAC dependente da hipófise, dependente da adrenocortical e iatrogênico. Esquematização do eixo corticotrófico em cães com hiperadrenocorticismo dependente da hipófise em (A), no qual a hipófise com neoplasia secreta excessivamente ACTH, com isso, ambas as glândulas adrenais recebem um estímulo exacerbado, o que as torna hiperplásicas, por consequência há um feedback negativo, o qual é irresponsivo pela hipófise. Já o esquema (B) representa o eixo do HAC dependente da adrenal, desse modo, uma das glândulas adrenais apresenta-se neoplásica e secreta o hormônio cortisol em excesso, o alto nível de cortisol realiza um feedback negativo no eixo, causando atrofia da glândula saudável, enquanto a adrenal doente não responde à inibição. Por fim, em (C) é representado o HAC iatrogênico, no qual o excesso de glicocorticoide exógeno realiza um feedback negativo em todo o eixo corticotrófico, promovendo a atrofia de ambas as adrenais.

\subsection{Hiperadrenocorticismo Iatrogênico}

A ocorrência do HAC de maneira iatrogênica consiste na administração excessiva de medicamentos à base de glicocorticoides e da exposição prolongada desse tratamento, com finalidade terapêutica de outras afecções, como alterações alérgicas e imunomediadas. Como há um elevado nível de cortisol circulante devido ao fármaco, o hormônio causa um feedback negativo no eixo corticotrófico, com isso, a produção de CRH e de ACTH é reduzida. Desse modo, a glândula adrenal é pouco estimulada, visto que o nível do cortisol já está suficiente, e, portanto, ocorre a atrofia bilateral da adrenal, como representado na Figura 2 (Marco, 2015; Nelson \& Couto, 2015).

\section{Fisiopatologia da hipercoagulabilidade secundária ao hiperadrenocorticismo}

A hipercoagulabilidade consiste no aumento da coagulação sanguínea, devido à elevação das proteínas hemostáticas, como os fatores de coagulação, ou pela redução da degradação dessas substâncias. Essa alteração favorece a ocorrência da trombose e, normalmente, acontece decorrente de anomalias metabólicas, inflamação, aumento da atividade plaquetária, aumento da ativação do fator de coagulação, deficiência de antitrombina e glomerulopatias (Zachary et al., 2012). Em animais sadios, o mecanismo de coagulação é iniciado por uma lesão vascular, que causa alterações imediatas nos vasos e, com isso, instaura o processo hemostático (Nelson \& Couto, 2015). Atualmente, é utilizado o modelo de coagulação baseado em superfícies celulares, que compreende quatro fases: iniciação, amplificação, propagação e finalização (Ferreira et al., 2010). Neste novo padrão, a hemostasia é iniciada através da exposição do fator tecidual (TF) na corrente sanguínea. $\mathrm{O}$ fator tecidual é um receptor de glicoproteínas encontrado nos tecidos extravasculares. Quando a lesão ocorre, as camadas mais profundas da parede dos vasos são rompidas e o TF exposto ao fluxo sanguíneo, iniciando as vias hemostáticas (Goddard, 2014). Nesse sentido, a fase de iniciação consiste em uma lesão endotelial que causa uma exposição do TF ao fluxo sanguíneo. Ao expor esse fator, ocorre uma interação entre ele e o fator VII da coagulação que está presente no plasma e, com isso, o fator IX e X são ativados. $\mathrm{O}$ fator $\mathrm{X}$ se liga ao fator $\mathrm{V}$ e 
transforma protrombina em trombina. A trombina formada é essencial para a próxima fase, a amplificação (Ferreira et al., 2010; Silva \& Melo, 2016).

A amplificação caracteriza-se pela ativação das plaquetas e pela ação da trombina. Quando o vaso é lesionado as plaquetas extravasam e se ligam ao colágeno situado próximo da ferida, formando um tampão plaquetário primário, o que consiste na hemostasia primária. Enquanto isso, a trombina formada na fase de iniciação interage com essas plaquetas, causando uma alteração na permeabilidade da superfície plaquetária, essa mudança libera substâncias quimiotáticas que atraem os fatores de coagulação para sua superfície. Além disso, a trombina possui a função de ativar o fator V e VIII da coagulação e, com a estimulação do fator VIII há a ação do fator de Von Willebrand (FvW) (Ferreira et al., 2010; Goddard, 2014). Segundo Takahira (2015), o FvW é uma glicoproteína que atua na adesão das plaquetas ao tecido subendotelial, sendo essencial para a estabilização e a manutenção do fator VIII da coagulação. Desse modo, inicia-se a formação de fibrina estável, fortalecendo o tampão plaquetário inicial, caracterizando a hemostasia secundária (Ferreira et al., 2010).

Após a formação do tampão plaquetário na fase de amplificação, ocorre a etapa de propagação. Esse período baseia-se no recrutamento de plaquetas para o local da injúria, e na formação de complexos tenase (participação do fator IX ativado e fator VIII ativado) e protrombinases (junção do fator X ativado e fator $\mathrm{V}$ ativado) na superfície das plaquetas ativadas. O complexo protrombinase é responsável pela grande produção de trombina, a qual irá fragmentar o fibrinogênio em monômeros de fibrina, consolidando o tampão plaquetário (Ferreira et al, 2010; Silva \& Melo, 2016). Por fim, ocorre a fase final desse processo, denominada etapa de finalização, que configura o controle da disseminação da coagulação. Isto é, o processo de hemostasia é limitado através de anticoagulantes naturais, como: proteína $\mathrm{C}$, proteína $\mathrm{S}$, inibidor da via do fator tecidual e antitrombina, visando evitar a formação de trombos e, consequentemente, a oclusão dos vasos sanguíneos (Ferreira et al., 2010; Nelson \& Couto, 2015).

A hipercoagulabilidade é favorecida pelo excesso crônico de cortisol, no entanto, a patogênese dessa relação ainda é pouco compreendida e caracteriza-se por um evento multifatorial (Park et al., 2013; Romão, 2012). Em vista disso, existem muitas hipóteses a respeito dessa associação, como: a estase venosa, lesão do epitélio vascular, perda glomerular de proteínas que diminui antitrombina III, aumento dos fatores de coagulação, aumento do fibrinogênio e plasminogênio (Ettinger et al., 2002).

Casonato et al. (1999) realizaram um estudo que associa o estado hipercoagulável ao aumento do fator de coagulação VIII e do fator de von Willebrand. Nessa pesquisa, foram selecionados vinte pacientes humanos com a Síndrome de Cushing, os quais possuíam aumento nos fatores de coagulação VIII e FvW. Esses pacientes foram submetidos a uma cirurgia terapêutica de retirada das neoplasias que causavam o aumento da liberação de cortisol. Após alguns meses da operação foi constatado uma normalidade nos valores de cortisol de alguns deles e, consequentemente, uma melhora nos fatores hemostáticos. Além disso, nos casos em que a cirurgia não efetivou a correção da liberação de glicocorticoides, os valores hemostáticos continuaram elevados. Desse modo, esses mesmos autores relacionaram a hipercoagulabilidade ao HAC devido as alterações quantitativas e qualitativas do fator VIII e do FvW.

Outra teoria para a característica hipercoagulável dos animais com HAC, é a que relaciona o alto nível de fibrinogênio à síndrome. Desse modo, Ambrosi et al. (2000) realizaram um estudo com onze pacientes que possuíam a doença e avaliaram um aumento de $20,9 \%$ na concentração de fibrinogênio em 55\% dos enfermos. Além de Ambrosi et al. (2000) outros pesquisadores como, Park et al. (2013) também relataram um aumento significativo de fibrinogênio nos seus pacientes em todos os momentos de sua pesquisa. Todavia, ao instituir o tratamento com trilostano, visando reduzir o cortisol circulante, obtiveram diminuição desses valores. Nesse sentido, há uma correlação entre o estado pro trombótico e a secreção de cortisol, visto que o fibrinogênio é importante para a formação da fibrina, a qual confere o tampão plaquetário. Sendo assim, o aumento do fibrinogênio contribui para a formação de trombos (Ambrosi et al., 2000).

Outro fundamento para a associação entre o aumento da coagulação e o HAC é a elevada pressão arterial característica dessa enfermidade. Afinal, a pressão alta causa um estresse nos vasos sanguíneos e, portanto, uma lesão endotelial. Com isso, há o estímulo dos fatores de ativação plaquetária, os quais liberam a cascata de coagulação (Park et al., 2013). De acordo com o estudo de Park et al. (2013), a pressão arterial foi avaliada durante o período de pesquisa em intervalos de três meses, nesse espaço de 
tempo foi verificado uma pressão arterial sistólica de 140-160 mmHg, que segundo Nelson e Couto (2015), é classificado como uma hipertensão arterial de grau moderado. Nesse mesmo estudo, Park et al. (2013) observaram a diminuição do tempo de protrombina (PT) e o aumento do número de plaquetas dos animais com hipercortisolismo quando comparados com os cães saudáveis. Esses valores não sofreram alterações relevantes mesmo após o início do tratamento, ou seja, continuaram elevados durante toda a pesquisa. Desse modo, os animais com hiperadrenocorticismo apresentaram a formação de um coágulo mais rápido e mais forte, configurando um marcador para o quadro de hipercoagulabilidade (Park et al., 2013). Outra possível explicação para o favorecimento de eventos trombóticos em cães com HAC está relacionada com a alteração renal causada pelo excesso do cortisol nesses pacientes. O hipercortisolismo promove uma alteração na filtração glomerular, o que contribui para a perda de proteína pela urina (Smets et al., 2010). Nesse sentido, cães que apresentam síndrome nefrótica possuem uma maior predisposição para eventos trombóticos devido à excreção urinária de antitrombina (Keyes et al., 1993). Afinal, como visto anteriormente, a antitrombina está relacionada com a fibrinólise, ou seja, atua na degradação dos fatores de coagulação (Romão, 2012). Portanto, o hipercortisolismo propicia um risco maior de quadros trombóticos visto a redução dos fatores anticoagulantes (Romão, 2012).

A fluidez sanguínea é determinada pela quantidade e qualidade das células do sangue e pela viscosidade do plasma sanguíneo (Windberger \& Bartholovitsch, 2004). O cortisol é reconhecido por ser um importante modulador de desenvolvimento de células sanguíneas, através do aumento da resposta dos precursores de eritrócitos à eritropoetina ou por estímulo direto da proliferação celular (Ámbrogio et al., 2014; Grafte-Faure et al., 1999). Sendo assim, em pacientes com HAC, é comum encontrar laboratorialmente eritrocitose, neutrofilia, monocitose e trombocitose (Feldman et al., 2015). Nesse contexto, Windberger e Bartholovitsch (2004) realizaram um estudo hemorreologico que avaliou a fluidez sanguínea no desenvolvimento do hiperadrenocorticismo.

Nessa pesquisa, os pacientes apresentaram aumento na viscosidade plasmática e agregação de hemácias, com isso o fluxo vascular tornou-se prejudicado. Esse quadro de estase venosa, segundo Feldman et al. (2015) contribuiu para um estado pró trombótico dos cães hiperadrenocorticoideos. Outra alteração celular decorrente do hipercortisolismo é a trombocitose, o mecanismo dessa relação ainda não é bem elucidado, porém, como visto anteriormente, o cortisol possui íntima ação na eritropoiese, sendo assim, possivelmente possui o mesmo efeito na trombocitopoiese (Park et al., 2013). Além dessa hipótese, os altos níveis de glicocorticoides endógenos causam o aumento das plaquetas, pois impedem a sua eliminação através da redução da fagocitose realizada por macrófagos (Prater \& Tvedten, 2004; Topper \& Welles, 2003 apud Marco et al., 2012). Segundo o estudo realizado por Marco et al. (2012), $44,5 \%$ dos cães submetidos à pesquisa apresentaram aumento plaquetário significativo, o que pode contribuir para um quadro pré-trombótico, visto que as plaquetas são importantes para a hemostasia primária e secundária, fornecendo fatores solúveis para a estabilização do coágulo. Além desses fatores, acredita-se que a obesidade, que é um dos sinais primários de hipercortisolismo, também favoreça um quadro pró trombótico (Arnaldi et al., 2003; Ettinger et al., 2002). Essa condição é resultante da liberação exacerbada pelo tecido adiposo de leptina, fator de necrose tumoral (TNF- $\alpha$ ), Inibidor 1 do ativador do plasminogênio (PAI-1) e fator tecidual (TF) em humanos e animais obesos, visto que há um excesso desse tecido nesses pacientes (Loskutoff \& Samad, 1998). A leptina é um hormônio produzido e secretado pelo tecido adiposo que induz a agregação plaquetária, segundo o estudo realizado com adolescentes obesos por Foschini et al. (2008), houve uma prevalência de 25,9\% de hiperleptinemia nos pacientes com obesidade quando comparados com jovens eutróficos. Além disso, foi verificado nesse mesmo estudo o aumento do número de plaquetas dos adolescentes com excesso de peso, essa estatística, como visto anteriormente, favoreceu a hipercoagulabilidade.

Em adição, outra relação da obesidade com o aumento da coagulação é decorrente da liberação em excesso do PAI-1. Essa proteína é responsável por inibir a fibrinólise, quando está aumentada na circulação compromete a limpeza da fibrina e, consequentemente, favorece a formação de trombos (Darvall et al., 2007; Loskutoff \& Samad, 1998). Nesse mesmo contexto, o tecido adiposo também é responsável por liberar interleucina 6 (IL-6) e TNF- $\alpha$, dessa maneira, a obesidade é considerada um estado crônico de inflamação, que causa um estresse oxidativo no endotélio, contribuindo para a agregação plaquetária (Darvall et al., 2007; Loskutoff \& Samad, 1998). Por fim, os fatores teciduais 
também contribuem para o aumento dos riscos cardiovasculares em cães obesos. Afinal, esse fator é encontrado em demasia nos animais com sobrepeso e propicia o início da cascata de coagulação, devido a ativação do fator VII presente na mesma (Loskutoff \& Samad, 1998).

\section{Manifestações clínicas e diagnóstico}

Animais que possuem hiperadrenocorticismo normalmente apresentam manifestações clínicas que refletem disfunções em vários sistemas (Peterson, 1984). Nesse contexto, o hiperadrenocorticismo deve ser diagnosticado através da união das informações do histórico do animal, das manifestações clínicas, dos exames físicos, dos testes laboratoriais de rotina, dos exames de imagem e hormonais (Peterson, 1984). As manifestações clínicas cardinais da afecção, normalmente, consistem em: polidipsia, poliúria, polifagia, dispneia, distensão abdominal, alopecia endócrina, obesidade e fraqueza muscular (Behrend et al., 2013; Nelson \& Couto, 2015; Peterson, 1984). Outras apresentações físicas podem aparecer decorrente do quadro de hipercortisolismo, como: letargia, hiperpigmentação e calcinose cutânea, comedões, pele afinada, incontinência urinária, atrofia testicular, anestro, frouxidão de ligamentos, paralisia facial, hepatomegalia, hipertensão arterial sistêmica e resistência insulínica (Behrend et al., 2013). Segundo Feldman et al. (2015), as manifestações clínicas não variam de acordo com a classificação do HAC, ou seja, se são dependentes da adrenal, da hipófise ou iatrogênico.

O estado hipercoagulável é pouco evidente clinicamente, porém está envolvido com a formação de trombos, que comumente afetam vasos pulmonares e, como consequência, provocam um desconforto e uma angústia respiratória no animal (Behrend et al., 2013; Klose et al., 2011). Os sinais clínicos desse tromboêmbolo que atinge o pulmão consistem em desconforto respiratório agudo, ortopneia e pulso jugular (Nelson \& Couto, 2015). Contudo, segundo Marco et al. (2012) e Dennis (1993) apesar da ausência de dispneia no momento da consulta, não é indicado descartar o comprometimento pulmonar, pois a trombose venosa periférica é assintomática no início e, posteriormente, pode acometer o leito pulmonar, causando desconforto respiratório agudo. Além do tromboembolismo pulmonar, os trombos formados nos cães com HAC também podem atingir a veia cava caudal, porta, esplênica e a artéria aorta (Delaforcade et al., 2019).

Além das alterações físicas, também é comum mudanças nos achados laboratoriais. Os animais com Síndrome de Cushing podem apresentar no leucograma leucocitose, eosinopenia, linfopenia e, no hemograma eritrocitose (Peterson, 1984). Já nos exames bioquímicos o principal achado é referente ao aumento de fosfatase alcalina presente em $85-90 \%$ dos pacientes com a afecção (Peterson, 1984). Outras anomalias encontradas no bioquímico são: hipercolesterolemia, hiperglicemia, hipertrigliceridemia, aumento de alanina aminotransferase (ALT), aumento de ácidos biliares e diminuição de ureia nitrogenada no sangue (Feldman et al., 2015). Na urinálise pode ser encontrado uma densidade abaixo do normal (menor que 1,010), além de possíveis sinais de infecção do trato urinário, como bacteriúria e hematúria (Peterson, 1984).

A suspeita da Síndrome pode ser baseada nos sinais clínicos e nos resultados dos exames de rotina, contudo, avaliações laboratoriais hormonais confirmam o diagnóstico e trazem informações sobre a origem da hipersecreção de cortisol (Peterson, 1984). Sendo assim, o teste de escolha para diagnosticar HAC é o de supressão por baixa dose de dexametasona, exceto quando há suspeita de HAC iatrogênico (Behrend et al., 2013). Esse teste consiste na administração intravenosa do glicocorticoide dexametasona, em uma dose baixa, $0,01 \mathrm{mg} / \mathrm{kg}$. A coleta de sangue é realizada em três momentos: antes da aplicação do medicamento, após quatro horas da aplicação e, por fim, oito horas depois da injeção de dexametasona (Kooistra \& Galac, 2012).

A interpretação desse exame é realizada a partir da análise do nível de cortisol presente no plasma sanguíneo coletado oito horas após a aplicação do glicocorticoide (Behrend et al., 2013). Se o aumento do cortisol, nesse intervalo de tempo, for superior a $40 \mathrm{nmol} / \mathrm{L}$ em cães com alterações físicas e bioquímicas que sinalizam a síndrome, é confirmado o HAC (Kooistra \& Galac, 2012). Além disso, é importante comparar o valor de cortisol apresentado na coleta realizada quatro horas após a administração do fármaco com o nível de cortisol presente na coleta feita antes do procedimento, se esse valor for menor que 50\% é possível que o HAC seja dependente da hipófise (Kooistra \& Galac, 2012). 
$\mathrm{O}$ teste de estimulação por ACTH é considerado o principal exame para diagnostico do HAC iatrogênico (Behrend et al., 2013). Ele consiste na determinação da concentração de cortisol sérico antes e uma hora depois da aplicação intravenosa ou intramuscular do hormônio ACTH sintético (Peterson, 1984). Em pacientes saudáveis, o aumento do cortisol é acima de $10 \mu \mathrm{g} / \mathrm{dL}$, entretanto, se o valor do cortisol após uma hora da administração do fármaco for maior que $20 \mu \mathrm{g} / \mathrm{dL}$ é indicativo de HAC (Peterson, 1984). Nos cães que possuem HAC iatrogênico, mesmo com o uso do ACTH, não ocorre aumento no nível de cortisol em relação ao valor basal (Nelson \& Couto, 2015; Peterson, 1984).

Outro método diagnóstico utilizado para diagnosticar o HAC é a relação entre o cortisol e a creatinina na urina, que promove uma leitura integrada da produção do glicocorticoide e, essa avaliação é refletida para as flutuações da concentração desse hormônio no sangue (Behrend et al., 2013). Este teste é rápido, não invasivo e possui uma alta precisão para diagnóstico do hipercortisolismo (Kooistra \& Galac, 2010). Para evitar efeitos colaterais do estresse, a coleta matinal da urina é realizada na casa do paciente por, pelo menos, 2 dias consecutivos (Kooistra \& Galac, 2010). Então, um pool é realizado e uma amostra única é enviada ao laboratório para realização e interpretação do teste através da divisão do valor de cortisol na urina pelo valor de creatinina, na maioria dos laboratórios a referência dessa relação é menor do que 15-20, não havendo unidade de medida. Sendo assim, animais que apresentam HAC possuem uma média mais elevada do que o padrão da referência (Peterson, 1984).

No entanto, nesse teste pode ocorrer resultados falso positivos, visto que algumas doenças não adrenais causam um estresse endógeno que aumenta a liberação de cortisol (Behrend et al., 2013; Peterson, 1984). Sendo assim, se o resultado do exame indicar positivo para o hipercortisolismo é importante associar os resultados obtidos do exame de supressão por baixa dose de dexametasona ou da estimulação por ACTH. Outra observação desse teste é que ele não consegue diferenciar a origem do hipercortisolismo (Peterson, 1984).

Após o resultado positivo nos exames de escolha para diagnóstico do hiperadrenocorticismo, segundo Behrend et al. (2013), é importante diferenciar se o HAC é devido uma alteração da hipófise ou da adrenal, pois os tratamentos e prognósticos podem variar. Nesse sentido, pode ser realizado o teste de supressão por alta dose de dexametasona, o qual distingue HAC dependente da adrenal e da hipófise (Behrend et al., 2013). Esse exame é realizado similarmente ao exame de supressão por baixa dose de dexametasona, exceto pela dose aplicada de medicamento, nesse caso $0,1 \mathrm{mg} / \mathrm{kg}$ (Nelson \& Couto, 2015).

Em pacientes com hipercortisolismo adrenal dependente a administração de qualquer dose de dexametasona não altera a secreção de cortisol, ou seja, o nível de cortisol continua elevado (Behrend et al., 2013). No entanto, se a origem do HAC for decorrente de alterações na hipófise, a dose alta do fármaco provoca uma supressão da secreção de ACTH em 75\% dos casos e, com isso, a concentração de cortisol reduz (Behrend et al., 2013). Nos pacientes que não ocorreram a supressão há uma chance de 50\% da origem da síndrome ser a adrenal, porém a outra metade pode ser devido afecção na hipófise, visto isso é necessário indicar testes adicionais para concluir a causa da doença (Peterson, 1984).

Outro exame que indica a origem do HAC é a mensuração da concentração endógena do hormônio adrenocorticotrófico. Normalmente, em cães com HAC dependente da hipófise o nível de ACTH no plasma pode estar elevado, enquanto nos cães com neoplasias na adrenal ou com HAC iatrogênico ocorre o oposto (Nelson \& Couto, 2015; Peterson, 1984).

Exames de imagem, como: radiografia abdominal, ultrassonografia do abdômen, tomografia computadorizada e ressonância magnética também são úteis para a diferenciação da origem do hiperadrenocorticismo (Peterson, 1984). Com a radiografia é possível identificar distensão abdominal, hepatomegalia, mineralização de brônquios e do interstício pulmonar e a presença de tumor na adrenal (Behrend et al., 2013). Já a ultrassonografia permite estimar a dimensão da glândula adrenal e a sua vascularização e, com isso, realiza a diferenciação do HAC com mais precisão do que os outros exames, sendo considerado o método de escolha para verificar a origem do hipercortisolismo. Por fim, a tomografia e a ressonância magnética, normalmente, são utilizadas para verificar a presença de um tumor de hipófise (Behrend et al., 2013; Peterson, 1984).

O diagnóstico do estado hiper coagulável nos animais com HAC é considerado desafiador, com isso, são usados exames como a tromboelastrografia (TEG) para avaliar a coagulação (Rose et al., 2013). A TEG analisa a coagulação como um todo, avaliando todas as etapas da hemostasia, por meio da 
mensuração da tensão exercida pela fibrina, da taxa de formação de coágulos e da força do mesmo (Grima et al., 2016; Klose et al., 2011). Esse exame é realizado a partir de uma amostra de sangue fresco total, que deve ser avaliado minutos após a coleta (Grima et al., 2016; Klose et al., 2011). O tromboelastograma gera três zonas que devem ser avaliadas, dentre elas a primeira fase refere-se a pré coagulação, ou seja, representa a formação de cadeias de fibrina. A segunda zona demonstra a formação do coágulo e, por fim, a terceira avalia a fibrinólise. Dentro dessas zonas são gerados alguns valores para serem analisados, conforme Figura 3. O (R) examina a via intrínseca, a qual está relacionada com os fatores VIII, IX, XI e XII da coagulação; o (K) consiste no tempo de formação do coágulo e a amplitude máxima (MA) demonstra a resistência do coágulo (Grima et al., 2016).

Segundo Rose et al. (2012), um cão pode ser considerado hipercoagulável quando apresenta alteração em algumas variáveis analisadas na TEG, como: aumento de MA, diminuição de $\mathrm{K}$ e/ou redução do ângulo $\alpha$. Nesse mesmo estudo, $66,6 \%$ dos animais com HAC avaliados apresentaram diminuição do K, $73,3 \%$ demonstraram um aumento no valor do MA; porém, apenas 6,7\% apresentaram aumento do ângulo $\alpha$. Outro estudo que também avaliou os parâmetros da TEG nos animais com HAC foi o de Park et al. (2013), o qual apresentaram resultados semelhantes ao de Rose et al. (2012) referente ao aumento da variável MA e a diminuição do K. No entanto, o ângulo $\alpha$ apresentou-se significativamente aumentado em suas análises. Pace et al. (2013), também verificaram através desse exame a presença de um quadro hipercoagulável nos animais com a afecção. Nessa pesquisa foram incluídos 17 cães com HAC, dentre eles 76,4\% apresentaram o valor do MA acima do padrão de referência e 17,6\% demostraram um aumento do ângulo $\alpha$.

Entretanto, no estudo realizado por Klose et al. (2011) não foi obtido uma relação entre cães com HAC e a hipercoagulabilidade. A maioria dos pacientes da pesquisa apresentaram um estado hipercoagulável independente do estado funcional da adrenal, entretanto, o valor de referência criado pelos pesquisadores foi baseado em cães jovens e saudáveis, o que é incompatível com a idade dos pacientes da pesquisa em si. Além disso, outras limitações foram identificadas no estudo devido ao baixo número de pacientes presentes no grupo de $\mathrm{HAC}$ e a falta de uma metodologia universal para a realização da TEG na Medicina Veterinária.

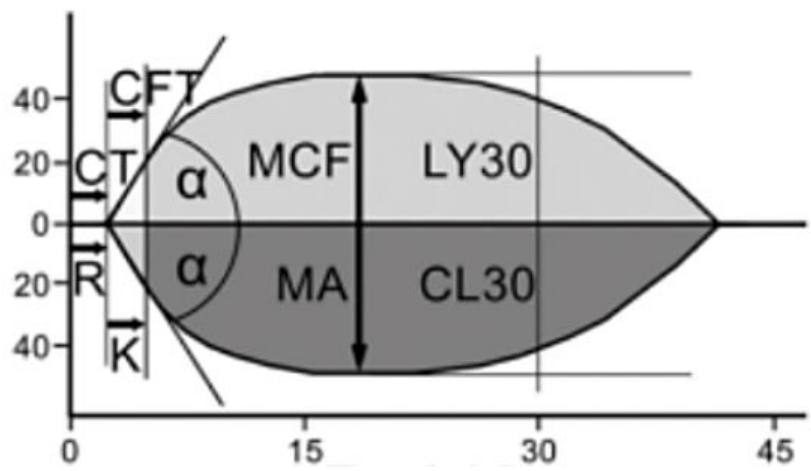

Figura 3. Traçado e variáveis que compõem o TEG e o ROTEM. Região com coloração cinza claro refere-se às variáveis presentes no ROTEM, enquanto na região cinza escura estão presentes os parâmetros avaliados na TEG. Fonte: Grima et al. (2016).

A tromboelastometria rotacional (ROTEM) é um exame bastante similar ao TEG quanto a finalidade e os parâmetros avaliados. Contudo, a técnica de aferição é diversificada entre esses dois testes, sendo assim, não é possível realizar uma comparação direta entre os dados obtidos dos exames (Goggs et al., 2014; Grima et al., 2016). As variáveis da ROTEM são: tempo para início da coagulação (CT), tempo de formação do coágulo (CFT), ângulo $\alpha$, força máxima do coágulo (MCF). A hipercoagulabilidade é considerada quando há um CT curto, associado ou não com a redução do CFT, aumento de MCF e/ou aumento do ângulo $\alpha$ (Coelho et al., 2014). Coelho et al. (2014) realizaram uma pesquisa avaliando a dinâmica de coagulação de 26 mulheres com HAC através da tromboelastometria rotacional. Foi a primeira pesquisa que realizou a investigação do estado coagulável de pessoas com Síndrome de Cushing por meio da ROTEM. A principal alteração encontrada, nesse estudo, entre as pacientes com a Síndrome e o grupo controle foi em relação à diminuição da variável CT, a qual indica uma formação 
mais rápida dos primeiros filamentos de fibrina, ou seja, evidencia uma alteração no início da formação do coágulo, apesar das outras etapas da coagulação não apresentarem alterações.

Além desses exames, o monitoramento da coagulação também pode ser feito através da avaliação do número de plaquetas, tempo sangramento de mucosas, tempo de protrombina, tromboplastina parcial ativada, concentração de fibrinogênio, avaliação do complexo trombina-antitrombina, mensuração do valor do fator VIII da coagulação, mensuração da antitrombina e realizar a equivalência de plaquetas e hematócrito (Grima et al., 2016; Pace et al., 2013). Esses testes podem realizar uma avaliação quantitativa da hemostasia e, desse modo, auxiliam no diagnóstico e na monitoração dos pacientes com alterações na coagulação (Grima et al., 2016).

Segundo o estudo realizado por Pace et al. (2013), foi observado em 31,2\% dos pacientes um aumento no número de plaquetas, $23,5 \%$ apresentaram tempo de tromboplastina parcial ativada prolongado, 17,6\% mostraram valores de fibrinogênio acima do padrão de referência, 52,9\% demonstraram aumento nos valores do complexo trombrina-antitrombina, além das alterações presentes nas variáveis da TEG citadas anteriormente. Sendo assim, 88,2\% dos animais desse estudo exibiram pelo menos uma alteração na coagulação e, com isso, verificou-se a importância da monitoração desses animais visto a tendência ao estado hipercoagulavel que apresentam.

Segundo Marco et al. (2012), é comum o aumento de fatores de procoagulação nos pacientes com hipercortisolismo. Nesse estudo notaram um aumento nos valores de fibrinogênio em $34 \%$ dos animais estudados e trombocitose em 44,5\%, o que de acordo com esses pesquisadores, favorece a formação de um coágulo estável. Além disso, Park et al. (2013) relataram uma redução no tempo de protrombina em $14,2 \%$ em relação ao valor padrão, contribuindo para a formação mais rápida do coágulo. Portanto, esses parâmetros são importantes para a suspeita precoce da formação de trombos (Marco et al., 2012).

\section{Tratamentos}

O tratamento do HAC deve ser instituído de acordo com a classificação da afecção, sendo assim, a terapia de escolha depende da etiologia do hipercortisolismo (Feldman et al., 2015). De acordo com Feldman et al. (2015), os cães com HAC dependente da hipófise, normalmente, são tratados com trilostano ou mitotano, esses fármacos possuem uma eficácia excelente para o tratamento do hipercortisolismo (Nelson \& Couto, 2015). O trilostano representa um inibidor competitivo da 3- $\beta$ hidroxiesteroide desidrogenase, essa enzima converte a pregnenolona em progesterona no córtex da adrenal, com isso, a inibição realizada pelo trilostano impede a síntese de cortisol pela glândula (Nelson \& Couto, 2015). A posologia indicada para esse medicamento é a administração uma vez ao dia (SID) na dose de 3-6 mg/kg (Augusto et al., 2012). No entanto, esse fármaco consegue controlar o nível de cortisol por aproximadamente 13 horas após a sua administração (Ettinger et al., 2002). Nesse sentido, Lemetayer \& Blois (2018) conduziram um estudo retrospectivo sobre o tratamento do HAC com trilostano e verificaram que a administração do fármaco duas vezes ao dia (BID) com uma dosagem de $0,5-1,0 \mathrm{mg} / \mathrm{kg}$ garante um tratamento efetivo e seguro.

Segundo Augusto et al. (2012), o uso da droga a cada 12 horas garante uma resolução mais rápida das manifestações clínicas; porém, consideraram essa diferença insignificante para a prática clínica. Contudo, Arenas et al. (2013) relataram em sua pesquisa que os cães tratados com o protocolo BID apresentaram uma melhora mais evidente dos sinais clínicos, além da dosagem ao final do dia com o protocolo BID ser menor do que a quantidade administrada uma vez ao dia.

Após o início do tratamento é indicado monitorar a resposta do organismo ao fármaco, em vista disso o teste de estimulação por ACTH é o exame mais indicado para essa etapa. Este deve ser realizado entre 10-14 dias depois da primeira administração de trilostano ou após a alteração de alguma dose, objetivando verificar se a supressão do cortisol não foi excessiva. Quando a dose se tornar estável a monitoração com o exame deve ser feita a cada 3-6 meses (Lemetayer \& Blois, 2018).

O mitotano, por sua vez, é um adrenocorcolítico que causa necrose no córtex da adrenal, seus efeitos à glândula são irreversíveis e, apesar de ser eficaz no tratamento do HAC dependente da hipófise, possui muitos efeitos colaterais, como: irritação gástrica, inapetência, sinais neurológicos e, quando a dose está em excesso, pode provocar hipocortisolismo e hipoaldosteronismo (Feldman et al., 2015; Nelson \& Couto, 2015). Esse fármaco deve ser administrado em duas fases: indução e manutenção. A fase de 
indução consiste na administração da droga com a dose de $30-50 \mathrm{mg} / \mathrm{kg} / \mathrm{dia}$ ou $25 \mathrm{mg} / \mathrm{kg} / \mathrm{BID}$ durante 7 a 10 dias ou até o animal apresentar sinais de hipocortisolismo, como: letargia, vômito, inapetência e apetite seletivo (Marco, 2015; Ettinger et al., 2002; Feldman et al., 2015; Nelson \& Couto, 2015).

Após apresentar os sinais característicos de baixo nível de cortisol, o animal deve ser submetido ao teste de estimulação por ACTH para verificar a eficácia do mitotano (Marco, 2015). O propósito desse medicamento é suscitar um hipocortisolismo relativo, sendo assim, depois da estimulação com ACTH o nível de cortisol deve estar entre 1-4 $\mu \mathrm{g} / \mathrm{dL}$ (Ettinger et al., 2002). Se o nível do hormônio não estiver inferior a $5 \mu \mathrm{g} / \mathrm{dL}$ é recomendado manter o mesmo protocolo inicial do tratamento e repetir o exame de estimulação por ACTH semanalmente. Na maioria dos pacientes com HAC, o valor de cortisol atinge o padrão esperado dentro de 5 a 10 dias (Nelson \& Couto, 2015).

A fase de manutenção é instituída quando o nível sérico de cortisol for inferior a $5 \mu \mathrm{g} / \mathrm{dL}$ e o cão apresenta-se hígido. Nessa etapa deve ser administrado $50 \mathrm{mg} / \mathrm{kg}$ de mitotano semanalmente, a qual pode ser fracionada nos dias da semana ou não. O teste de estimulação por ACTH deve ser realizado após 3 meses do início da fase de manutenção e posteriormente a cada seis meses. Se o teste apresentar uma concentração de cortisol abaixo de $2 \mu \mathrm{g} / \mathrm{dL}$ e o animal não mostrar apatia, vômito e inapetência a dose do medicamento deve ser reduzida para $25 \mathrm{mg} / \mathrm{kg} / \mathrm{semana}$. No entanto, se o paciente demonstrar sinais de hipoadrenocorticismo o tratamento deve ser interrompido (Marco, 2015; Ettinger et al. 2002; Nelson \& Couto, 2015).

Segundo Barker et al. (2005), a escolha do fármaco não influencia na sobrevivência dos animais com HAC dependente da hipófise, ou seja, não há diferença significativa no tempo de vida entre os pacientes tratados com o protocolo de trilostano ou mitotano. Para esse estudo foram selecionados 148 cães com HAC de origem hipofisária, diagnosticados com aproximadamente 10 anos de idade. $\mathrm{O}$ mitotano foi instituído em 25 cães, os quais apresentaram uma sobrevida de aproximadamente 708 dias, enquanto o trilostano foi administrado em 123 animais e a média de sobrevivência foi de 662 dias. Nesse sentido, Barker et al. (2005) verificaram que o fator mais influente para reduzir a vida dos cães com HAC foi o diagnóstico tardio da afecção e a presença de obesidade nos animais submetidos ao tratamento.

Para os animais com HAC dependente da adrenal é indicado como tratamento a remoção cirúrgica do tumor, conhecida como adrenalectomia (Marco, 2015). No entanto, não é possível utilizar essa técnica quando o animal apresenta metástase ou riscos anestésicos devido a doenças concomitantes (Nelson \& Couto, 2015). Além disso, a complicação mais preocupante da adrenalectomia é o tromboembolismo, o qual normalmente ocorre dentro das 24 horas após a cirurgia e, é considerado fatal (Nelson \& Couto, 2015). Sendo assim, o tratamento com mitotano e trilostano também é recomendado para esses pacientes (Marco, 2015).

Nesses cães com HAC dependente da adrenal a escolha do medicamento também não influencia significativamente o tempo de sobrevida deles (Helm et al., 2011). Na pesquisa realizada por Helm et al. (2011), foi verificado que animais tratados com mitotano possuem um tempo de vida médio de 102 dias, enquanto os animais que utilizaram o trilostano como fármaco de escolha sobreviveram por aproximadamente 353 dias. Contudo, não consideraram esses valores relevantes para estabelecer uma relação com a droga e o tempo de vida dos pacientes, sendo a principal causa de diminuição da sobrevivência a presença de metástase nos cães selecionados.

Segundo Park et al. (2013) o quadro hiper coagulável dos animais com HAC não apresenta melhora com o controle da secreção de cortisol, ou seja, mesmo se o nível do hormônio estiver dentro do padrão de referência a maioria dos marcadores de coagulação permanecem alterados, sendo o fibrinogênio o único indicador que sofreu uma redução significativa nesse estudo. Os valores que representam a hipercoagulabilidade pela TEG não apresentam melhora com o tratamento do hipercortisolismo e com a, consequente, melhora das manifestações clínicas nos animais (Kol et al., 2013). Isso pode sugerir um efeito prolongado do cortisol no organismo ou que algum fator da patogênese da hipercoagulabilidade no hipercortisolismo ainda precisa ser melhor identificado (Kol et al., 2013). Park et al. (2013) explicaram esse achado pela duração do efeito do trilostano, visto que esse medicamento possui ação por no máximo 18 horas no organismo. Desse modo, animais que receberam a droga uma vez ao dia ficaram expostos a um período significativo de hipercortisolismo na circulação sanguínea. Isso pode ser suficiente para a permanência da hipercoagulabilidade, apesar da melhora das manifestações clínicas. 
Além dessa explicação, esses mesmos pesquisadores acreditavam que o cortisol gerava uma alteração endotelial irreversível nesses animais e, com isso, o quadro de coagulação não obtinha melhora com o controle do hormônio.

Por outro lado, no estudo de Casonato et al. (1999), os pacientes submetidos a cirurgia terapêutica para o hipercortisolismo apresentaram, logo após o procedimento, uma redução insignificante dos marcadores de coagulação. Todavia, após 12 meses do tratamento os valores hemostáticos estavam normalizados, enquanto, os pacientes que não conseguiram uma eficiência do tratamento através da cirurgia continuaram com os sinais de hipercoagulação presentes. Portanto, esses resultados sugeriram que é necessário um longo período para a melhora dos efeitos metabólicos que o cortisol provoca nas células. Como citado anteriormente, o cortisol pode causar danos irreversíveis no endotélio e seu efeito é prolongado no organismo, o que dificulta a resolução do quadro hiper coagulável mesmo com o controle do hipercortisolismo. Sendo assim, a prevenção dessa anormalidade representa a melhor solução, uma vez que o prognóstico das afecções trombóticas é de reservado a grave (Boscaro et al., 2002; Dennis, 1993). Nesse sentido, é indicado para humanos o uso de heparina nos pacientes diagnosticados com o aumento da coagulação secundária ao hipercortisolismo (Arnaldi et al., 2003).

Segundo Boscaro et al. (2002), o uso de drogas anticoagulantes de maneira profilática no pós-operatório de pacientes com HAC reduziu a morbidade e a mortalidade por eventos trombóticos para, respectivamente, $6,0 \%$ e $0,4 \%$. O estudo realizado por esses autores utilizou 307 pacientes humanos com Síndrome de Cushing, os quais foram divididos em 2 grupos. O grupo um abrangia 75 pacientes e não recebia heparina preventivamente. Já o grupo dois possuía 232 pacientes e era administrado heparina profilaticamente no pós-operatório. A diferença de eventos trombóticos foi significativa entre os grupos, $20 \%$ do grupo um apresentou complicações tromboembólicas, dos quais 10,7\% vieram a óbito. Enquanto no grupo dois apenas $6 \%$ desenvolveu tromboembolismo e a mortalidade dentro desse grupo foi de $0,4 \%$.

Nos cães com HAC as drogas antitrombóticas não devem ser consideradas quando não há afecção concomitante que agrave o risco da formação de trombos, conforme a Tabela 1, ou quando os exames que confirmem esse estado hipercoagulável não tenham sido realizados (Delaforcade et al., 2019; Nelson \& Couto, 2015; Sharp et al., 2019). O objetivo do tratamento consistiu em prevenir a extensão e formação de novos trombos e impedir a anastomose entre veias e artérias (Nelson \& Couto, 2015).

Tabela 1. Condições que aumentam os riscos de tromboembolismo pulmonar.

\begin{tabular}{ll}
\hline Condições que predispõem a ocorrência do tromboembolismo pulmonar & \\
\hline Dirofilariose & Insuficiência cardíaca congestiva \\
Síndrome nefrótica & Anormalidade hemostática primária \\
Anemia hemolítica imunomediada & Trauma \\
Hiperadrenocorticismo & Hipotireoidismo \\
Pancreatite & Síndrome de choque \\
Neoplasias & Cetoacidose diabética \\
Sepse & Endocardite bacteriana \\
\hline
\end{tabular}

Fonte: Adaptado Keyes et al. (1993).

Sharp et al. (2019) realizaram uma aplicação clínica do consenso sobre o uso racional de antitrombóticos em veterinária, através do relato do caso de um cão com hiperadrenocorticismo que desenvolveu um quadro de pancreatite. Esse animal, portanto, possuía duas afecções que possivelmente podiam causar o aumento da coagulação, segundo o consenso ao qual se baseavam (Delaforcade et al., 2019). Sendo assim, o animal foi submetido à exames e apresentou sinais de hipercoagulabilidade sendo, então, instituído um tratamento leve para a coagulopatia pelo uso de anticoagulantes.

Segundo Sharp et al. (2019), quando a pancreatite está associada ao HAC é mais comum a ocorrência de trombos venosos. Sendo assim, os medicamentos mais eficazes para impedir o tromboembolismo venoso são os anticoagulantes quando comparados aos antiplaquetários (Goggs et al., 2019). Nesse sentido, no caso apresentado por Sharp et al. (2019), os medicamentos de escolha poderiam ser: heparina de baixo peso molecular, heparina não fracionada ou rivaroxabana.

A heparina é indicada para conter a extensão do trombo e prevenir a formação de novos coágulos, essa droga não realiza a trombólise e age ativando a antitrombina e estimulando a liberação de inibidores 
de fatores teciduais (Nelson \& Couto, 2015). A heparina não fracionada pode ser administrada via intravenosa com a dosagem de $100 \mathrm{U} / \mathrm{kg}$ bolus, seguida de 480-900 U/kg/SID em infusão continua. Esse fármaco também pode ser administrado pela via subcutânea com a dose de 150-300 U/kg a cada 6 horas, essa posologia pode ser ajustada conforme o monitoramento do animal. Esse medicamento não deve ser manipulado oralmente, pois não há relatos que confirmem a sua eficácia por essa via (Blais et al., 2019). A heparina de baixo peso molecular também é uma alternativa segura para controlar o tromboembolismo, essa variante inibe pouco a trombina e, com isso, reduz o risco de hemorragia como efeito colateral (Nelson \& Couto, 2015). Dentro desse tipo de heparina há a daltaparina e a enoxaparina, cujas dosagens subcutâneas são 100-175 U/ $/ \mathrm{kg}$ a cada oito horas e $0,8 \mathrm{mg} / \mathrm{kg}$ a cada seis horas, respectivamente (Blais et al., 2019).

O rivaroxabana consiste em um inibidor direto do fator Xa da coagulação e é considerado um fármaco seguro e bem tolerado pelos animais (Blais et al., 2019; Sharp et al., 2019). Esse medicamento deve ser administrado via oral com a dosagem de $1-2 \mathrm{mg} / \mathrm{kg} / \mathrm{SID}$ e, normalmente, ele é preferível pela facilidade da manipulação via oral (Blais et al., 2019; Goggs et al., 2019). No entanto, ainda são necessários estudos mais aprofundados sobre a eficácia desse medicamento e a sua posologia adequada para a tromboprofilaxia (Blais et al., 2019).

Por fim, a terapia antiplaquetária objetiva melhorar o fluxo sanguíneo e reduzir a formação de agregados plaquetários, sendo mais indicada para tromboembolismos arteriais, como: o tromboembolismo pulmonar e aórtico (Goggs et al., 2019; Nelson \& Couto, 2015). A aspirina e o clopidogrel são fármacos de escolha para esse tipo de terapia, ambos possuem baixo risco para hemorragia e um monitoramento menos rigoroso (Nelson \& Couto et al., 2014). A dosagem da aspirina é de $0,5 \mathrm{mg} / \mathrm{kg} / \mathrm{BID}$ e deve ser administrada por via oral, já o clopidogrel deve ser fornecido uma vez ao dia 1-3mg/kg também por via oral (Blais et al., 2019; Nelson \& Couto, 2015). Além disso, para a obtenção de uma resposta mais rápida do clopidogrel no organismo pode ser realizado uma única dose oral de $4-10 \mathrm{mg} / \mathrm{kg}$ (Blais et al., 2019).

\section{Considerações finais}

O HAC é uma das principais endocrinopatias que acomete cães de meia idade a idosos, essa afecção é caracterizada pela secreção excessiva de cortisol, o qual pode ocorrer em dependência do estímulo advindo da hipófise, da adrenal ou de maneira iatrogênica. Esse atributo confere alterações em diferentes órgãos e sistemas, isto é, o hipercortisolismo é responsável por inúmeras manifestações clínicas, cujo excesso prolongado desse hormônio pode gerar complicações fatais ao animal.

Como abordado nessa revisão literária a hipercoagulabilidade secundária ao excesso de cortisol é pouco compreendida pelos autores, os quais consideram para tal relação diferentes etiologias, como: aumento dos fatores de coagulação; lesão endotelial consequente da elevada pressão arterial; trombocitose; estase venosa; entre outras.

Visto as vastas possibilidades para o aumento da coagulação nesses animais, o diagnóstico precoce tanto do HAC como da hipercoagulabilidade são relevantes. Nesse sentido, existem diversos testes que podem diagnosticar o hiperadrenocorticismo, dentre eles o considerado nível ouro é o de supressão por baixa dose de dexametasona. Já para o diagnóstico da hipercoagulabilidade os exames TEG e ROTEM são bastantes úteis para avaliar a hemostasia como um todo, entretanto, testes mais simples como: tempo de protrombina, concentração de fibrinogênio e fatores de coagulação, contagem plaquetária, entre outros, também são pertinentes.

O tratamento do HAC não se mostrou muito eficaz para a correção da hipercoagulabilidade, uma vez que, hipoteticamente, o cortisol confere danos irreversíveis ao endotélio e possui efeito prolongado no organismo. Sendo assim, apesar do seu controle necessita de um tempo para cessar seus efeitos. Entretanto, essas teorias não foram bem elucidadas, de modo que é recomendado o início de uma terapia de suporte com anticoagulantes para os cães que apresentaram predisposição para a formação de trombos. Através da presente revisão literária, portanto, foi possível compreender que são necessários mais estudos para compreender as causas da hipercoagulabilidade consequente do hipercortisolismo, bem como, a sua possível prevenção e tratamento. 


\section{Referências bibliográficas}

Ambrogio, A. G., Martin, M., Ascoli, P., \& Cavagnini, F. (2014). Gender-dependent changes in haematological parameters in patients with Cushing's disease before and after remission. European Journal of Endocrinology, 170(3), 393-400. https://doi.org/10.1530/EJE-13-0824.

Ambrosi, B., Sartorio, A., Pizzocaro, A., Passini, E., Bottasso, B., \& Federici, A. (2000). Evaluation of haemostatic and fibrinolytic markers in patients with Cushing s syndrome and in patients with adrenal incidentaloma. Experimental and Clinical Endocrinology \& Diabetes, 108(4), 294-298. https://doi.org/10.1055/s-2000-8000.

Arenas, C., Melian, C., \& Perez-Alenza, M. D. (2013). Evaluation of 2 trilostane protocols for the treatment of canine pituitary-dependent hyperadrenocorticism: Twice daily versus once daily. Journal of Veterinary Internal Medicine, 27(6), 1478-1485. https://doi.org/10.1111/jvim.12207.

Arnaldi, G., Angeli, A., Atkinson, A. B., Bertagna, X., Cavagnini, F., Chrousos, G. P., Fava, G. A., Findling, J. W., Gaillard, R. C., \& Grossman, A. B. (2003). Diagnosis and complications of Cushing's syndrome: a consensus statement. The Journal of Clinical Endocrinology \& Metabolism, 88(12), 5593-5602. https://doi.org/10.1210/jc.2003-030871.

Augusto, M., Burden, A., Neiger, R., \& Ramsey, I. (2012). A comparison of once and twice daily administration of trilostane to dogs with hyperadrenocorticism. Tierärztliche Praxis Ausgabe K: Kleintiere/Heimtiere, 40(6), 415-424. https://doi.org/10.1055/s-0038-1623672.

Barker, E. N., Campbell, S., Tebb, A. J., Neiger, R., Herrtage, M. E., Reid, S. W. J., \& Ramsey, I. K. (2005). A comparison of the survival times of dogs treated with mitotane or trilostane for pituitarydependent hyperadrenocorticism. Journal of Veterinary Internal Medicine, 19(6), 810-815. https://doi.org/10.1111/j.1939-1676.2005.tb02769.x.

Behrend, E. N., Kooistra, H. S., Nelson, R., Reusch, C. E., \& Scott-Moncrieff, J. C. (2013). Diagnosis of spontaneous canine hyperadrenocorticism: 2012 ACVIM consensus statement (small animal). Journal of Veterinary Internal Medicine, 27(6), 1292-1304. https://doi.org/10.1111/jvim. 12192.

Blais, M., Bianco, D., Goggs, R., Lynch, A. M., Palmer, L., Ralph, A., \& Sharp, C. R. (2019). Consensus on the Rational Use of Antithrombotics in Veterinary Critical Care (CURATIVE): Domain 3Defining antithrombotic protocols. Journal of Veterinary Emergency and Critical Care, 29(1), 6074. https://doi.org/10.1111/vec.12794.

Boscaro, M., Sonino, N., Scarda, A., Barzon, L., Fallo, F., Sartori, M. T., Patrassi, G. M., \& Girolami, A. (2002). Anticoagulant prophylaxis markedly reduces thromboembolic complications in Cushing's syndrome. The Journal of Clinical Endocrinology \& Metabolism, 87(8), 3662-3666. https://doi.org/10.1210/jcem.87.8.8703.

Casonato, A., Pontara, E., Boscaro, M., Sonino, N., Sartorello, F., Ferasin, S., \& Girolami, A. (1999). Abnormalities of von Willebrand factor are also part of the prothrombotic state of Cushing's syndrome. Blood Coagulation \& Fibrinolysis, 10(3), 145-151. https://doi.org/10.1097/00001721-199904000-00006.

Coelho, M. C. A., Vieira Neto, L., Kasuki, L., Wildemberg, L. E., Santos, C. V., Castro, G., Gouvêa, G., Veloso, O. C. G., Gadelha, T., \& Gadelha, M. R. (2014). Rotation thromboelastometry and the hypercoagulable state in C ushing's syndrome. Clinical Endocrinology, 81(5), 657-664. https://doi.org/10.1111/cen.12491

Darvall, K. A. L., Sam, R. C., Silverman, S. H., Bradbury, A. W., \& Adam, D. J. (2007). Obesity and thrombosis. European Journal of Vascular and Endovascular Surgery, 33(2), 223-233. https://doi.org/10.1016/j.ejvs.2006.10.006.

Delaforcade, A., Bacek, L., Blais, M., Goggs, R., Lynch, A., \& Rozanski, E. (2019). Consensus on the rational use of antithrombotics in veterinary critical care (CURATIVE): domain 1-defining populations at risk. Journal of Veterinary Emergency and Critical Care, 29(1), 37-48. https://doi.org/10.1111/vec.12797.

Dennis, J. S. (1993). Clinical features of canine pulmonary thromboembolism. The Compendium on Continuing Education for the Practicing Veterinarian (USA), 15(12), 1595-1603.

Ettinger, S. J., Fedlman, E. C., \& Taibo, R. A. (2002). Tratado de medicina interna veterinaria: enfermedades del perro y el gato. Manole. 
Feldman, E. C., Nelson, R. W., Reusch, C., \& Scott-Moncrieff, J. C. (2014). Canine and feline endocrinology. Elsevier Health Sciences.

Ferreira, C. N., Sousa, M. O., Dusse, L. M. S., \& Carvalho, M. G. (2010). O novo modelo da cascata de coagulação baseado nas superfícies celulares e suas implicações. Revista Brasileira de Hematologia e Hemoterapia, 32, 416-421. https://doi.org/10.1590/S1516-84842010000500016.

Foschini, D., Santos, R. V. T., Prado, W. L., Piano, A., Lofrano, M. C., Martins, A. C., Carnier, J., Caranti, D. A., Sanches, P. L., \& Tock, L. (2008). Platelet and leptin in obese adolescents. Jornal de Pediatria, 84, 516-521. https://doi.org/10.1590/S0021-75572008000700008.

Goddard, A. (2014). The Risk of thrombosis in veterinary patients.

Goggs, R., Bacek, L., Bianco, D., Koenigshof, A., \& Li, R. (2019). American College of Veterinary Emergency and Critical Care (ACVECC) Consensus on the Rational Use of Antithrombotics in Veterinary Critical Care (CURATIVE) guidelines: Small animal. Journal of Veterinary Emergency and Critical Care, 29(1), 12-36. https://doi.org/10.1111/vec.12801.

Goggs, Robert, Brainard, B., Delaforcade, A. M., Flatland, B., Hanel, R., McMichael, M., \& Wiinberg, B. (2014). Partnership on rotational viscoelastic test standardization (PROVETS): evidence-based guidelines on rotational viscoelastic assays in veterinary medicine. Journal of Veterinary Emergency and Critical Care, 24(1), 1-22. https://doi.org/10.1111/vec.12144.

Grafte-Faure, S., Leveque, C., Vasse, M., Soria, C., Norris, V., \& Vannier, J. (1999). Effects of glucocorticoids and mineralocorticoids on proliferation and maturation of human peripheral blood stem cells. American Journal of Hematology, 62(2), 65-73. https://doi.org/10.1002/(SICI)10968652(199910)62:2\%3C65::AID-AJH1\%3E3.0.CO;2-G.

Grima, T., Santori, I., Alcânara, M. A., Sibuti, C., Ribas, J., \& Bolfer, L. (2016). Viscoelastografia na medicina veterinária intensiva. Veterinary \& Science, 32-39.

Helm, J. R., McLauchlan, G., Boden, L. A., Frowde, P. E., Collings, A. J., Tebb, A. J., Elwood, C. M., Herrtage, M. E., Parkin, T. D. H., \& Ramsey, I. K. (2011). A comparison of factors that influence survival in dogs with adrenal-dependent hyperadrenocorticism treated with mitotane or trilostane. Journal of Veterinary Internal Medicine, 25(2), 251-260. https://doi.org/10.1111/j.1939-1676.2011.0696.x.

Jericó, M. M., Kogika, M. M., \& Andrade Neto, J. P. (2015). Tratado de medicina interna de cães e gatos. Guanabara Koogan.

Keyes, M. L., Rush, J. E., \& Knowles, K. E. (1993). Pulmonary thromboembolism in dogs. Journal of Veterinary Emergency and Critical Care, 3(1), 23-32. https://doi.org/10.1111/j.14764431.1993.tb00100.x.

Klose, T. C., Creevy, K. E., \& Brainard, B. M. (2011). Evaluation of coagulation status in dogs with naturally occurring canine hyperadrenocorticism. Journal of Veterinary Emergency and Critical Care, 21(6), 625-632. https://doi.org/10.1111/j.1476-4431.2011.00696.x.

Kol, A., Nelson, R. W., Gosselin, R. C., \& Borjesson, D. L. (2013). Characterization of thrombelastography over time in dogs with hyperadrenocorticism. The Veterinary Journal, 197(3), 675-681. https://doi.org/10.1016/j.tvj1.2013.05.047.

Köning, H. E., \& Liebich, H. G. (2011). Anatomia dos animais domésticos texto e atlas colorido. Editora Artmed.

Kooistra, H. S., \& Galac, S. (2012). Recent advances in the diagnosis of Cushing's syndrome in dogs. Topics in Companion Animal Medicine, 27(1), 21-24. https://doi.org/10.1053/j.tcam.2012.06.001.

Lemetayer, J., \& Blois, S. (2018). Update on the use of trilostane in dogs. The Canadian Veterinary Journal, 59(4), 397. https://doi.org/Lemetayer, J.; Blois, S. Uptade on the us

Loskutoff, D. J., \& Samad, F. (1998). The adipocyte and hemostatic balance in obesity: studies of PAI1. Arteriosclerosis, Thrombosis, and Vascular Biology, 18(1), 1-6. https://doi.org/10.1161/01.ATV.18.1.1.

Marco, V. (2015). Doenças do sistema endócrino e do metabolismo. In M. Jericó, J. P. Neto, \& M. Kogika (Eds.), Tratado de medicina interna de cães e gatos. Guanabara - Koogan.

Marco, Viviani, Winkel, V. M., \& Martorelli, C. R. (2012). Estudo da hipercoagulabilidade sanguínea em 45 cães com hiperadrenocorticismo endógeno, por meio da avaliação da frequência de trombocitose, hiperfibrinogenemia e hipertensão arterial. Clínica Veterinária, 17(96), 44-50. 
Nelson, R. W., \& Couto, C. G. (2015). Medicina interna de pequenos animais (Issue 1). Elsevier Editora.

Pace, S. L., Creevy, K. E., Krimer, P. M., \& Brainard, B. M. (2013). Assessment of coagulation and potential biochemical markers for hypercoagulability in canine hyperadrenocorticism. Journal of Veterinary Internal Medicine, 27(5), 1113-1120. https://doi.org/10.1111/jvim.12159.

Park, F., Blois, A., Abrams-Ogg, A., Wood, R., Allen, D., Nykamp, S. \& Downie, A. (2013). Hypercoagulability and ACTH-dependent hyperadrenocorticism in dogs. Journal of Veterinary Internal Medicine, v. 27, n. 5, p. 1136-1142, 2013. DOI: https://doi.org/10.1111/jvim.12162

Peterson, M. E. (2007). Diagnosis of hyperadrenocorticism in dogs. Clinical Techniques in Small Animal Practice, 22(1), 2-11.

Rijnberk, A., \& Kooistra, H. S. (2010). Clinical endocrinology of dogs and cats: an illustrated text. Schlütersche.

Romão, F. G. Análise do perfil hemostático e do risco tromboembólico em cães submetidos ao tratamento com prednisona. 2012. Dissertação (Mestrado em Medicina Veterinária). Universidade Estadual Paulista, Botucatu, 2012.

Rose, L., Dunn, M. E., \& Bedard, C. (2013). Effect of canine hyperadrenocorticism on coagulation parameters. Journal of Veterinary Internal Medicine, 27(1), 207-211. https://doi.org/10.1111/jvim.12005.

Sharp, C. R., Goggs, R., Blais, M., Brainard, B. M., Chan, D. L., DeLaforcade, A. M., \& Rozanski, E. (2019). Clinical application of the American College of Veterinary Emergency and Critical Care (ACVECC) Consensus on the Rational Use of Antithrombotics in Veterinary Critical Care (CURATIVE) guidelines to small animal cases. Journal of Veterinary Emergency and Critical Care, 29(2), 121-131. https://doi.org/10.1111/vec.12801.

Silva, R. D. R., \& Melo, E. M. (2016). A atual teoria da coagulação baseada em superfícies celulares. Saúde \& Ciência Em Ação, 2(1), 79-92. https://doi.org/10.1590/S1516-84842010000500016

Smets, P., Meyer, E., Maddens, B., \& Daminet, S. (2010). Cushing's syndrome, glucocorticoids and the kidney. General and Comparative Endocrinology, 169(1), 1-10. https://doi.org/10.1016/j.ygcen.2010.07.004.

Takahira, R. K. (2015). Hemostasia normal. In M. Jericó, J. P. Neto, \& M. Kogika (Eds.), Tratado de medicina interna de cães e gatos. Guanabara Koogan.

Waki, M. F., Ribas Martorelli, C., Erdmann Mosko, P., \& Kogika, M. M. (2010). Classificação em estágios da doença renal crônica em cães e gatos: abordagem clínica, laboratorial e terapêutica. Ciência Rural, 40(10), 1-9.

Windberger, U., \& Bartholovitsch, A. (2004). Hemorheology in spontaneous animal endocrinopathies. Clinical Hemorheology and Microcirculation, 31(3), 207-215.

Zachary, J. F., McGavin, D., \& McGavin, M. D. (2012). Bases da patologia em veterinária. Elsevier Brasil.

Histórico do artigo:

Recebido: 27 de maio de 2021

Aprovado: 3 de julho de 2021
Licenciamento: Este artigo é publicado na modalidade Acesso Aberto sob a licença Creative Commons Atribuição 4.0 (CC-BY 4.0), a qual permite uso irrestrito, distribuição, reprodução em qualquer meio, desde que o autor e a fonte sejam devidamente creditados. 